\title{
Enhanced Radiation of Near-Inertial Energy by Frontal Vertical Circulations
}

\author{
LEIF N. THOMAS \\ Department of Earth System Science, Stanford University, Stanford, California
}

(Manuscript received 31 January 2019, in final form 14 July 2019)

\begin{abstract}
Near-inertial waves (NIWs) radiate energy out of the mixed layer when they develop small lateral scales. Refraction of these waves by gradients in planetary and vertical vorticity has traditionally been invoked to explain this phenomenon. Here, a new mechanism for the enhancement of NIW radiation is described involving the interaction of NIWs with vertical circulations at fronts undergoing frontogenesis. Frontal vertical circulations drive a Doppler shift that is proportional to the wave's vertical wavenumber $m$ and that changes sign across a front, inducing large lateral differences in wave phase within a few inertial periods. Theory predicts that the process should generate a vertical energy flux that varies inversely with $m$ in contrast to the $\mathrm{m}^{-3}$ dependence expected from refraction. As a consequence, high-mode NIWs are much more effective at radiating energy when fronts and their vertical circulation are present. Numerical simulations initialized with fronts, an array of eddies that drive frontogenesis, and NIWs of various modes are used to test the theory. In the simulations, the interaction of the NIWs with the frontal vertical circulations generates wave beams that radiate down from the fronts. The resultant downward energy flux varies with $m$ following the theoretical scaling laws. In the beams, the Eulerian frequency is inertial within a few percent, yet the waves' potential and kinetic energies are comparable, thus indicating a superinertial intrinsic frequency. The downshift in Eulerian frequency from the intrinsic frequency is due to horizontal advection of the waves by the eddies.
\end{abstract}

\section{Introduction}

It is thought that the mixing that sustains the abyssal stratification and circulation derives a significant fraction of its energy from wind-driven near-inertial waves (NIWs; Alford et al. 2016). Generated at large lateral scales set by the wind's footprint on the ocean, these NIWs are inherently inefficient at radiating energy down to the abyss without a shift to smaller wavelengths. Hence the conundrum: how can the lateral gradients in near-inertial motions initially set by the winds be sharpened so as to hasten the propagation of NIW energy into the ocean interior?

The prevailing theory for how the lateral scales of NIWs are sharpened relies on gradients in the Coriolis parameter $f$ or the vertical vorticity $\bar{\zeta}$ of the balanced flow field. The idea is that the frequency of NIWs is modulated horizontally by variability in $f$ and $\bar{\zeta}$, which in turn generates lateral differences in wave phase and hence a horizontal wavenumber $k_{h}$. This mechanism, which has been referred to as the $\beta$ effect (e.g.,

\footnotetext{
Corresponding author: Leif N. Thomas, leift@stanford.edu
}

D'Asaro 1989) and NIW refraction in the theory of Young and Ben-Jelloul (1997), results in a linear increase of $k_{h}$ with time at a rate that is proportional to the lateral gradients of $f$ or $\bar{\zeta}$ (D'Asaro 1995; van Meurs 1998). Once generated by refraction, the horizontal wavenumber can be further strengthened by lateral strain in the balanced flow, until dispersion ultimately limits the growth of $k_{h}$ (Rocha et al. 2018). These wavemean flow mechanisms only consider background flows with horizontal velocities. Vertical velocities in background flows are ignored because they are either absent or their effects on the waves are negligible. Neither of these conditions strictly hold for NIWs at ocean fronts and this has implications for the evolution of their horizontal wavenumber and vertical propagation.

Ocean fronts are often accompanied by vertical circulations (e.g., Pollard and Regier 1992; Rudnick 1996). Driven by a variety of processes, frontal vertical motions typically form in response to a disruption of the thermal wind balance, alternate sign across a front, and have magnitudes ranging between 1 and $100 \mathrm{~m}$ day $^{-1}$ (Thomas et al. 2010). If an inertia-gravity wave were 
embedded in a front, these vertical motions would advect its flow perturbations. This could be especially disruptive to the evolution of NIWs since they are characterized by strong vertical shears. To estimate the effect of this vertical advection on a NIW, a Doppler shift

$$
\omega_{v \text { Dop }}=\bar{w} m
$$

(where $\bar{w}$ is the vertical velocity of the background flow and $m$ is a vertical wavenumber characterizing the depth dependence of the wave) can be calculated and compared to its intrinsic frequency. For example, for a vertical circulation of $10 \mathrm{mday}^{-1}$ acting on a wave with a vertical wavelength of $\mathscr{O}(100) \mathrm{m}$, typical of a low-mode NIW, the Doppler shift is $\omega_{v \text { Dop }} \sim 7 \times$ $10^{-6} \mathrm{~s}^{-1}$, which in midlatitudes is nearly $10 \%$ of $f$. High-mode NIWs will experience even larger Doppler shifts. These adjustments to the NIWs' frequency are comparable to frequency shifts associated with vorticity in mesoscale and submesoscale flows, suggesting that it might not be justified to neglect vertical advection while retaining the effects of wave refraction by vorticity in a background flow with fronts.

Apart from being a significant fraction of $f$, the Doppler shift (1) changes sign across a front and this could affect the evolution of a NIW's lateral structure and vertical propagation. As $\bar{w}$ switches from upwelling to downwelling across a front, a NIW should accumulate lateral differences in phase as the wave oscillates at different frequencies on either side of the frontal interface. This would sharpen the wave's horizontal scale and increase $k_{h}$. Consequently, the vertical component of the group velocity, which for NIWs is well approximated by

$$
c_{g, z} \approx-\frac{N^{2} k_{h}^{2}}{f m^{3}}
$$

( $N$ is the buoyancy frequency), would increase in magnitude, enhancing the radiation of NIW energy in the vertical.

Motivated by the scaling arguments for the Doppler shift (1) and its potential to decrease the lateral scale of NIWs, the objective of this article is to quantify how the interaction of NIWs with frontal vertical circulations impacts the vertical radiation of NIWs. To this end both analytical methods (e.g., section 2) and numerical simulations (e.g., section 3 ) will be used to illustrate the phenomenon and highlight the dependence of vertical radiation on the key parameters of the frontal flow and wave field. A particular emphasis will be placed on quantifying the dependence on the vertical wavenumber of the
NIWs given the sensitivity of the group velocity (2) and Doppler shift to $m$, and the potential implications for the dynamics of low- and high-mode NIWs will be highlighted.

\section{Evolution of a NIW's horizontal wavenumber in a vertical circulation}

The aim is to determine the conditions for which the Doppler shift (1) is the dominant mean flow interaction that a NIW experiences and to derive an expression for the time evolution of the horizontal wavenumber $k_{h}$ that results under these conditions. To do so, it will be assumed a priori that these conditions are met and a solution for $k_{h}$ will be derived. The solution will then be contrasted to the horizontal wavenumber that results from refraction to determine when vertical Doppler shifting dominates over refraction. When the vertical Doppler shift is the leadingorder wave-mean flow interaction, and the waves are linear (e.g., when a Rossby number based on the wave's velocity and length scale is much less than one) the equations governing the NIWs are

$$
\begin{gathered}
\frac{\partial u_{w}}{\partial t}-f v_{w}+\bar{w} \frac{\partial u_{w}}{\partial z}=0, \\
\frac{\partial v_{w}}{\partial t}+f u_{w}+\bar{w} \frac{\partial v_{w}}{\partial z}=0 .
\end{gathered}
$$

where $\left(u_{w}, v_{w}\right)$ is the horizontal velocity associated with the NIW, and it has been assumed that the lateral wavelength of the waves is very long and the frequency of the waves is very close to $f$ so that pressure gradients and horizontal advection play a negligible role in the dynamics. It will also be assumed for simplicity that the background flow and waves are two dimensional (i.e., invariant in the $x$ direction). It should be realized that the vertical velocity of the background flow in (3) and (4) can be a function of space and time, potentially making it challenging to solve these equations. However, if the temporal and vertical spatial scales of $\bar{w}$ are long compared to those of the waves, then the method of multiple scales can be used to solve the equations. That is, new independent variables can be introduced, $\eta$ and $\tau$, that characterize the slow vertical and temporal variations of the background flow, respectively, that is, $\bar{w}=\bar{w}(y, \eta, \tau)$. If the NIW is defined by a single vertical wavenumber $m$, then the leadingorder solution to (3) and (4) takes the form $u_{w}=$ $A(y, \eta, \tau) e^{i \Phi}+$ c.c., where $A$ is an amplitude function and

$$
\Phi=m z-f t-m \int \bar{w} d t
$$


describes the wave's phase. Defining a horizontal wavenumber as the lateral gradient of the wave's phase, yields the key result that differential vertical motions integrated over time generate a horizontal wavenumber:

$$
k_{h, v \mathrm{Dop}} \equiv \frac{\partial \Phi}{\partial y}=-m \int \frac{\partial \bar{w}}{\partial y} d t .
$$

This mechanism can be contrasted to refraction, which for a vorticity field that varies only in the $y$ direction results in a horizontal wavenumber (van Meurs 1998)

$$
k_{h, \text { ref }}=-\frac{1}{2} \frac{\partial \bar{\zeta}}{\partial y} t .
$$

Scaling the two expressions (6) and (7) and taking their ratio yields the following nondimensional parameter

$$
\left[\frac{k_{h, v \mathrm{Dop}}}{k_{h, \mathrm{ref}}}\right] \sim \frac{W L_{b}}{U H_{w}},
$$

where the square brackets denote a scaling, $U$ and $W$ are scalings for the horizontal and vertical components of the background flow, $L_{b}$ scales the lateral variations of the background flow, and $H_{w}$ is a measure of the wave's vertical wavelength. If the background flow is quasigeostrophic, then $W=\varepsilon U\left(H_{b} / L_{b}\right)$, where $H_{b}$ is the vertical scale of the background flow and $\varepsilon=U / f L_{b}$ is the Rossby number (Pedlosky 1987). In this limit the ratio (8) becomes

$$
\left[\frac{k_{h, v \text { Dop }}}{k_{h, \text { ref }}}\right]_{\mathrm{QG}} \sim \frac{\varepsilon}{\delta},
$$

where

$$
\delta=\frac{H_{w}}{H_{b}}
$$

is a nondimensional parameter that compares the vertical scales of the waves and the background flow. It therefore follows that vertical Doppler shifting will produce smaller horizontal wave scales than refraction, and will be the dominant wave-mean flow interaction for NIWs, when $\varepsilon / \delta>1$. This condition is met when the vertical scale of the NIWs is small enough so that $H_{w}<\varepsilon H_{b}$. For currents with higher Rossby numbers (such as submesoscale flows), the appropriate scaling for the vertical velocity is $W=$ $U\left(H_{b} / L_{b}\right)$ and $(8)$ becomes

$$
\left[\frac{k_{h, v \mathrm{Dop}}}{k_{h, \mathrm{ref}}}\right]_{\mathrm{SG}} \sim \frac{1}{\delta},
$$

in which case vertical Doppler shifting dominates when $H_{w}<H_{b}$. Regardless of the choice of scaling for the vertical velocity, however, it is clear that NIWs with smaller vertical scales tend to be more strongly affected by vertical Doppler shifting over refraction.

Apart from the relative scales of the two mechanisms, the essential difference between (6) and (7) is that $k_{h, v \text { Dop }}$ depends on the vertical wavenumber of the wave, suggesting that high-mode NIWs are more susceptible to differential vertical motions, unlike refraction, which acts indiscriminately on both the low and high modes. This has important implications for the vertical radiation of NIW energy. Since $c_{g, z} \propto k_{h}^{2} / m^{3}$, the generation of a horizontal wavenumber by the Doppler shift should result in a vertical energy flux that follows an $m^{-1}$ power law, in contrast to $\mathrm{m}^{-3}$ for refraction. As a consequence, high-mode NIWs should be much more effective at radiating energy when they interact with frontal vertical circulations versus vertical vorticity gradients. To test this hypothesis, idealized numerical simulations were performed and are described in the next section.

\section{Numerical simulations}

The simulations are configured with three flow components: fronts, an array of barotropic eddies, and a NIW. The eddies provide vorticity and a strain field that drives frontogenesis. During frontogenesis, a frontal vertical circulation is generated, the strength of which depends on the strain rate, stratification, and the frontal lateral density gradient (Hoskins et al. 1978; Thomas et al. 2008). This vertical circulation increases rapidly with time as the fronts intensify but in a manner that can readily be controlled by varying the strain and the lateral and vertically gradients of the density field at the start of each simulation. A NIW with no initial lateral variations is added to this system and the development of a horizontal wavenumber through interactions with the frontal vertical circulation or the vorticity can be evaluated. The simulations were run using the Regional Ocean Modeling System (ROMS), which solves the fully three-dimensional, nonlinear, primitive equations of motion (Shchepetkin and McWilliams 2005). Using a hydrostatic model like ROMS to study the dynamics of NIWs is fully justified since the aspect ratio of the waves $H_{w} / L_{w}$ is quite small. The fronts used in the simulations similarly have small aspect ratios and therefore their dynamics is captured by the primitive equations as well. 


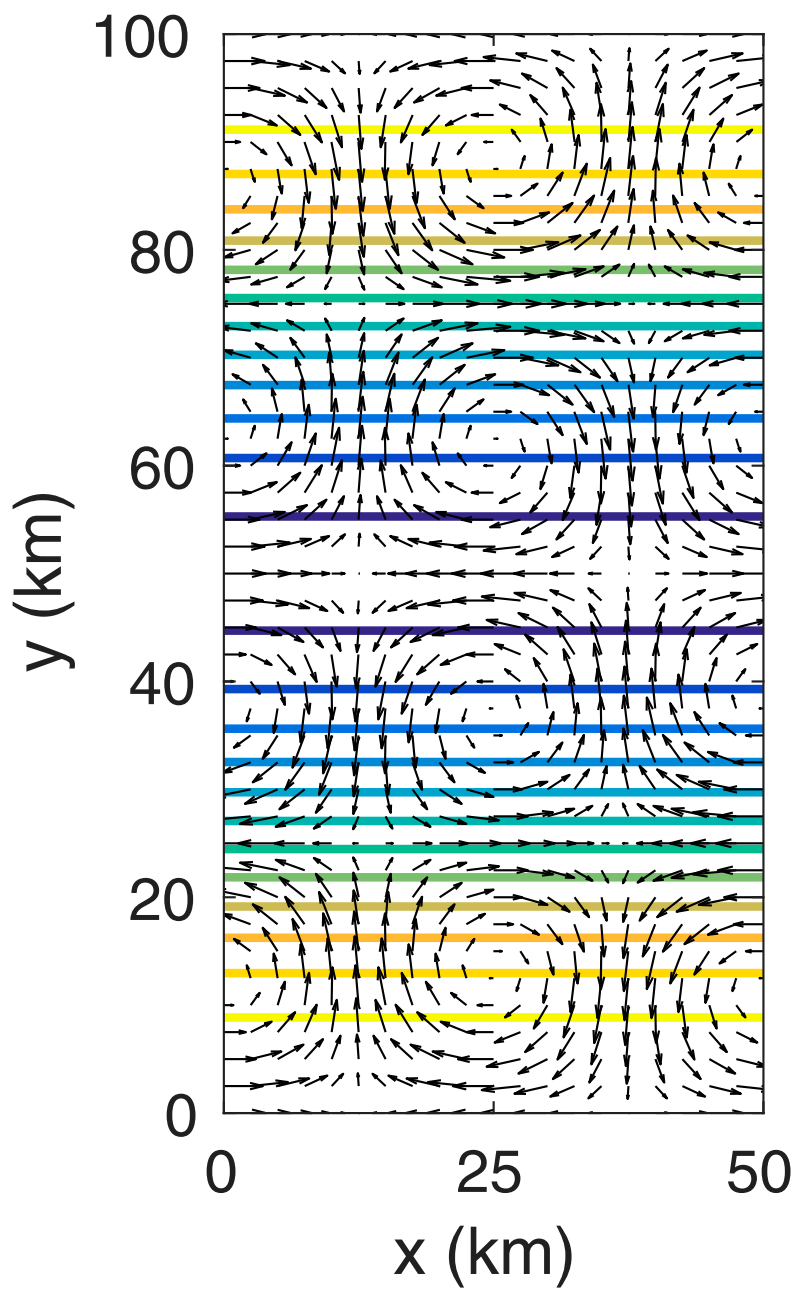

FIG. 1. Flow of barotropic eddies (vectors) and the initial surface buoyancy field (contours) used in simulation RUN1. The largest velocity vector corresponds to $0.054 \mathrm{~m} \mathrm{~s}^{-1}$, and the contour interval of the buoyancy field is $2.5 \times 10^{-4} \mathrm{~m} \mathrm{~s}^{-2}$, with lighter shades indicating larger buoyancy.

\section{a. Frontal flow and eddies}

The particular functional form of the initial density and eddy flow fields is described in the appendix and is illustrated in Figs. 1 and 2 (top panel) for one of the numerical simulations (i.e., RUN1, see Table 1). There are two fronts centered at $y=25$ and $75 \mathrm{~km}$. The fronts are surface intensified and are in geostrophic balance with a velocity in the $x$ direction (Fig. 2, top panel). The stratification is reduced near the surface, and increases with depth. The fronts are initially weak, more specifically they are characterized by a Richardson number, $\mathrm{Ri}_{o}=N^{2} f^{2} /(\partial \bar{b} / \partial y)^{2}$, that is initially larger than 10 (e.g., Table 1). However, in regions of frontogenetic strain (such as near $x=12.5 \mathrm{~km}$ and $y=25$ and $75 \mathrm{~km}$ ) the fronts rapidly
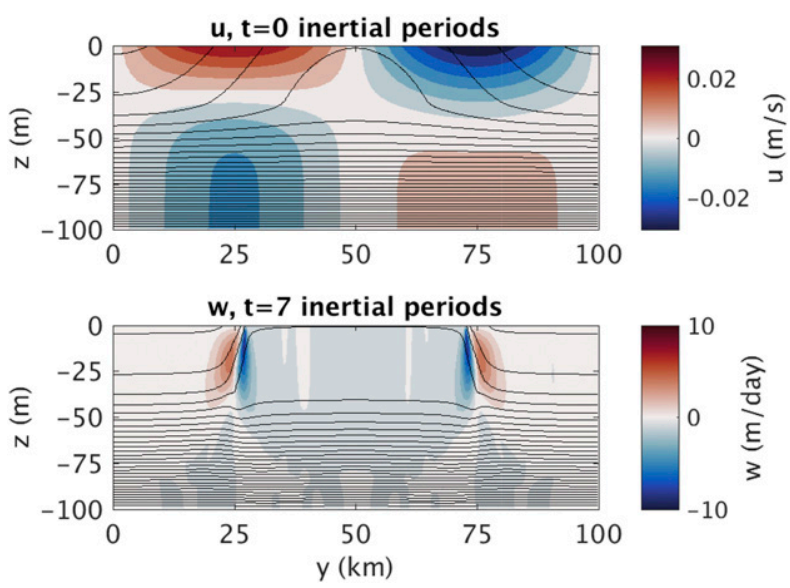

FIG. 2. (top) Vertical cross section at $x=12.5 \mathrm{~km}$ of the initial condition for RUN1 of the buoyancy (contours) and the $x$ component of the velocity (color). (bottom) Cross section of buoyancy (contours) and the vertical velocity at 7 inertial periods into the simulation illustrating the sharpening of the fronts by eddydriven strain and the generation of a frontal vertical circulation with downwelling and upwelling on the dense and light sides of the fronts, respectively. The contour interval of buoyancy is $9.8 \times 10^{-4} \mathrm{~m} \mathrm{~s}^{-2}$.

strengthen with time and the Richardson number in the center of the front drops to values close to one in less than 10 inertial periods. This is the case even though the maximum strain rate of the eddies, $\alpha_{o}$ is relatively modest, that is, $\alpha_{o} / f<0.1$. As the front sharpens, a vertical circulation is induced with velocities approaching $10 \mathrm{~m} \mathrm{day}^{-1}$ (Fig. 2, bottom panel). Consistent with the theory of frontogenesis, the secondary circulation is thermally direct, and thus characterized by downwelling and upwelling on the dense and light sides of the front, respectively.

\section{b. Simulations with idealized wave forms}

The frontal flow has all the elements to significantly modify the lateral structure of a NIW through the Doppler shift (1) and this was investigated by adding a NIW to the initial condition. The NIW has no horizontal wavenumber initially, and consists of a plane wave with vertical wavenumber $m$ and amplitude that is surface intensified, see, (A5) and Fig. 3 (top panel). The vertical wavenumber is discretized in terms of the depth of the domain, $H=100 \mathrm{~m}$, that is, $m=2 \pi n / H$, where the integer $n$ is the mode number. The NIW is surface intensified so as to highlight the downward radiation of wave energy. The form of the wave is highly idealized and was chosen because using a well-defined vertical wavenumber facilitates quantifying the dependence of the NIW evolution on $m$. Simulations with wave forms more representative of the ocean will be described in section $3 \mathrm{c}$. 
TABLE 1. Parameters used to construct the initial conditions for the numerical simulations (see the appendix for the definitions of the variables).

\begin{tabular}{lccccc}
\hline \hline Experiment & $S_{o}^{2}\left(\mathrm{~s}^{-2}\right)$ & $f\left(\mathrm{~s}^{-1}\right)$ & $\alpha_{o}\left(\mathrm{~s}^{-1}\right)$ & $N_{\mathrm{ml}}^{2}\left(\mathrm{~s}^{-2}\right)$ & $3 \times 10^{-5}$ \\
\hline RUN1 & $1 \times 10^{-7}$ & $1 \times 10^{-4}$ & $6 \times 10^{-6}$ & $3 \times 10^{-5}$ & $\mathrm{Ri}_{o}=N_{\mathrm{ml}}^{2} f^{2} / S_{o}^{4}$ \\
RUN1-NF & 0 & $1 \times 10^{-4}$ & $6 \times 10^{-6}$ & $3 \times 10^{-5}$ & 30 \\
RUN2 & $1 \times 10^{-7}$ & $1 \times 10^{-4}$ & $1 \times 10^{-5}$ & $3 \times 10^{-5}$ & 30 \\
RUN2-NF & 0 & $1 \times 10^{-4}$ & $1 \times 10^{-5}$ & $3 \times 10^{-5}$ & 30 \\
RUN3 & $1 \times 10^{-7}$ & $1 \times 10^{-4}$ & $1.4 \times 10^{-5}$ & $3 \times 10^{-5}$ \\
RUN3-NF & 0 & $1 \times 10^{-4}$ & $1.4 \times 10^{-5}$ & $\infty$ \\
\hline
\end{tabular}

To isolate the effects of the vertical Doppler shift from refraction, two sets of simulations are performed with the same stratification and barotropic eddy field, but with and without fronts (e.g., Table 1). The simulations without fronts have a variable vorticity field that can modify the horizontal wavenumber of the waves through refraction, but the Doppler shift is not active. Therefore by comparing the wave evolution in the two sets of simulations the effects of the vertical circulation on the evolution of the wave field can be quantified. For each set of experiments the mode number of the wave is varied between 1 and 7 .

The evolution of a NIW with $n=6$ in a background flow with and without fronts is strikingly different (cf. Figs. 3 and 4$){ }^{1}$ In the former, wave phase lines tilt in the proximity of the fronts, generating significant horizontal variability in the wave field within a few inertial periods, unlike the simulations without fronts. Near fronts, phase lines are distorted into a " $z$ "-like shape, tilting against isopycnals in the center of a front and parallel to isopycnals on its flanks. This shape appears to reflect advection by the frontal vertical circulation and the changing sign of $\partial \bar{w} / \partial y$ across the front. The shrinking of the NIW's horizontal scale clearly enhances wave radiation, as evidenced by the internal wave beams that form beneath the fronts by 7 inertial periods and the deficit in wave kinetic energy (KE) in the fronts left in their wake (e.g., Fig. 3, bottom panel). In contrast, in the run without fronts, very little wave energy is evident in the stratified interior by this time (e.g., Fig. 4, bottom panel).

To test if the sharpening of NIW scales seen in the simulations with fronts is ultimately attributable to the frontal vertical circulation, a wavelet analysis was performed to infer the distribution of scales present

\footnotetext{
${ }^{1}$ In the figures and subsequent analyses, wave quantities are isolated from frontal ageostrophic circulations and balanced flows by subtracting the output from simulations run without waves (but with the same eddy and density fields) from those with a NIW included in the initial conditions.
}

in the wave field near the fronts, and the results were compared to the theoretical prediction for the evolution of the wavevector by the Doppler shift, that is, (6). To this end, a continuous wavelet transform of the NIW $y$ component of the velocity $v_{w}$ was calculated, that is, $\mathscr{W}_{v}(y, a)=(1 / \sqrt{a}) \int \psi[(y-\xi) / a] v_{w}(\xi) d \xi$, using a complex Morlet wavelet of the form

$$
\psi(s)=\frac{1}{\sqrt{\pi}} \exp (i 3 \pi s) \exp \left(-s^{2}\right),
$$

where $a$ is the scale of the wavelet. The wavelet transform was evaluated along $x=12.5 \mathrm{~km}$ and at various depths and times. The square of the amplitude of the wavelet transform, $|\mathscr{\mathscr { V }}|_{v}^{2}$, at $z=-25 \mathrm{~m}$ and $t=5.1 \mathrm{in}$ ertial periods for RUN1 illustrates how the finest scales in the wave field are found near the fronts at $y=25$ and $75 \mathrm{~km}$ (e.g., Fig. 5a). A Hovmöller diagram of the wavelet transform at the center of the fronts quantifies how the lateral variability in the wave field evolves (Fig. 5b). A range of scales develops, but the finest scales with the largest wavenumbers track the nearexponential growth of the theoretical prediction (6) (magenta line in Fig. 5). The theoretical prediction was calculated using a vertical wavenumber $m=12 \pi / H$ corresponding to the mode number $n=6$ of the experiment and using $\partial \bar{w} / \partial y$ evaluated at $y=25 \mathrm{~km}$ and $z=-25 \mathrm{~m}$ from the simulation run without the NIW. This differential vertical motion and hence (6) increases nearly exponentially with time due to the strain and the frontogenesis it induces. The theory also predicts the dominant wavenumber of the wave beam that emanates from the front (e.g., Fig. 5c). By 7 inertial periods the wavelet transform at $z=-50 \mathrm{~m}$ and $y=27.5 \mathrm{~km}$ has a peak near a wavenumber of $k_{h}=3 \times 10^{-3} \mathrm{rad} \mathrm{m}^{-1}$ that is intersected by the theoretical prediction (6).

The wave beam that forms underneath the front has fairly steep phase lines, suggesting that the wave is superinertial, however, time series of wave properties within the beam reveal oscillations with a frequency close to $f$ (Fig. 6). To infer the intrinsic frequency of the wave more quantitatively, the slope of phase lines 

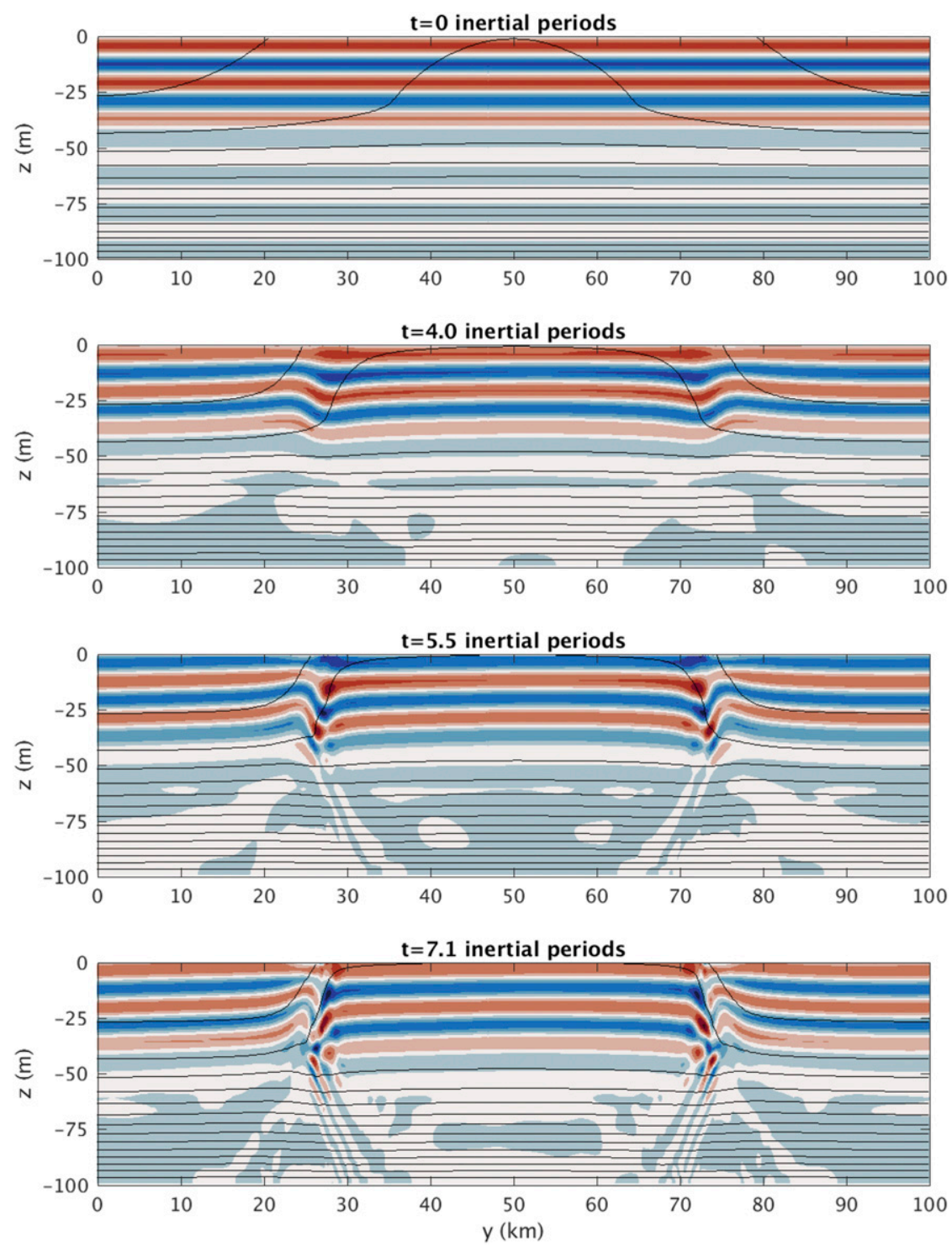

FIG. 3. Evolution of a NIW with $n=6$ for RUN1 with fronts undergoing frontogenesis. The $y$ component of the wave velocity $v_{w}$ is plotted in color, and isopycnals are contoured for a cross section at $x=12.5 \mathrm{~km}$. As the vertical circulation of the frontal ageostrophic secondary circulation strengthens, it tilts wave phase lines upward, causing the waves to radiate downward and leaving a deficit of wave $\mathrm{KE}$ in the fronts. The maximum wave velocity is $0.01 \mathrm{~m} \mathrm{~s}^{-1}$.

within the beam were compared to wave rays of various intrinsic frequencies. The rays were calculated using the classical dispersion relation for inertia-gravity waves that does not account for modifications by the vorticity and baroclinicity of the background flows which are negligible beneath the mixed layer and for superinertial waves. A ray with intrinsic frequency $\omega_{i}=1.7 f$ closely follows the slope of the wave beam (Fig. 6a). Further evidence of the superinertial nature of the waves is the noncircular velocity hodographs (Fig. 6b) and significant wave buoyancy anomalies
(Fig. 6c) in the beam. According to classical theory for plane inertia-gravity waves, the strength of the wave buoyancy anomaly relative to its velocity (which is related to the ratio of the potential to kinetic energy in the wave) is given by the quantity

$$
\frac{\left|b_{w}\right|}{N\left|v_{w}\right|}=\sqrt{1-\frac{f^{2}}{\omega_{i}^{2}}},
$$

which for an intrinsic frequency $\omega=1.7 f$ is equal to 0.8. A time series of $b_{w} / N$ within the beam is 

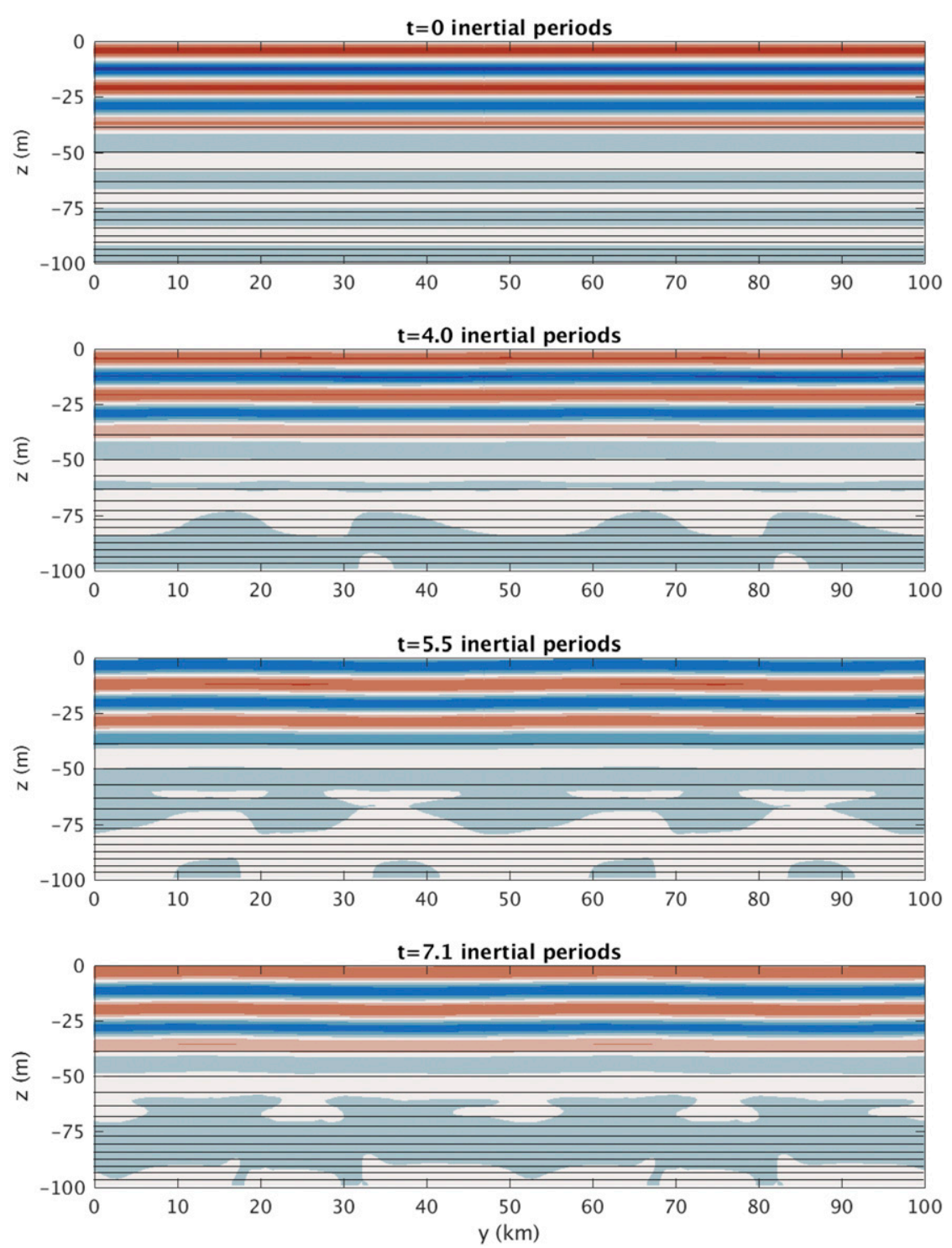

FIG. 4. As in Fig. 3, but for RUN1-NF, a simulation with the same eddy field, but without the fronts. Very little wave energy is radiated downward in this simulation.

characterized by oscillations that are $10 \%-20 \%$ weaker in magnitude than $v_{w}$, consistent with an intrinsic frequency of $\omega=1.7 f$ (e.g., Fig. 6c). In contrast, the Eulerian frequency, $\omega_{E}=0.97 f$, calculated by performing a harmonic analysis on the time series is much closer to inertial. The difference between the Eulerian and intrinsic frequencies is attributable to a Doppler shift associated with the barotropic, confluent, meridional flow field associated with the eddies, that is $\omega_{E}-\omega_{i} \approx \bar{v} k_{h}$. At the location where the time series shown in Fig. $6 \mathrm{c}$ was made, $\bar{v} \approx-0.03 \mathrm{~m} \mathrm{~s}^{-1}$, which given the estimate of the horizontal wavenumber in the beam from the wavelet analysis, $k_{h}=$ $3 \times 10^{-3} \mathrm{rad} \mathrm{m}^{-1}$, yields a Doppler shift, $\bar{v} k_{h} \sim-0.9 f$, that can explain the difference between Eulerian and intrinsic frequencies.

\section{1) DEPENDENCE ON VERTICAL WAVENUMBER}

In simulations run with NIWs spanning a range of mode numbers $n$ but with the same background flow as in RUN1, wave beams emanate from the fronts and result in a peak in the wavelet transform, similar to what is seen in Fig. 5c, however the dominant wavenumber and timing of the peak varies with $n$ (e.g., Fig. 7). The dominant wavenumber increases with $n$, consistent with the theoretical prediction (6). The arrival of the wave beam at $z=-50 \mathrm{~m}, y=27.5 \mathrm{~km}$ is generally earlier for the low modes even though their 


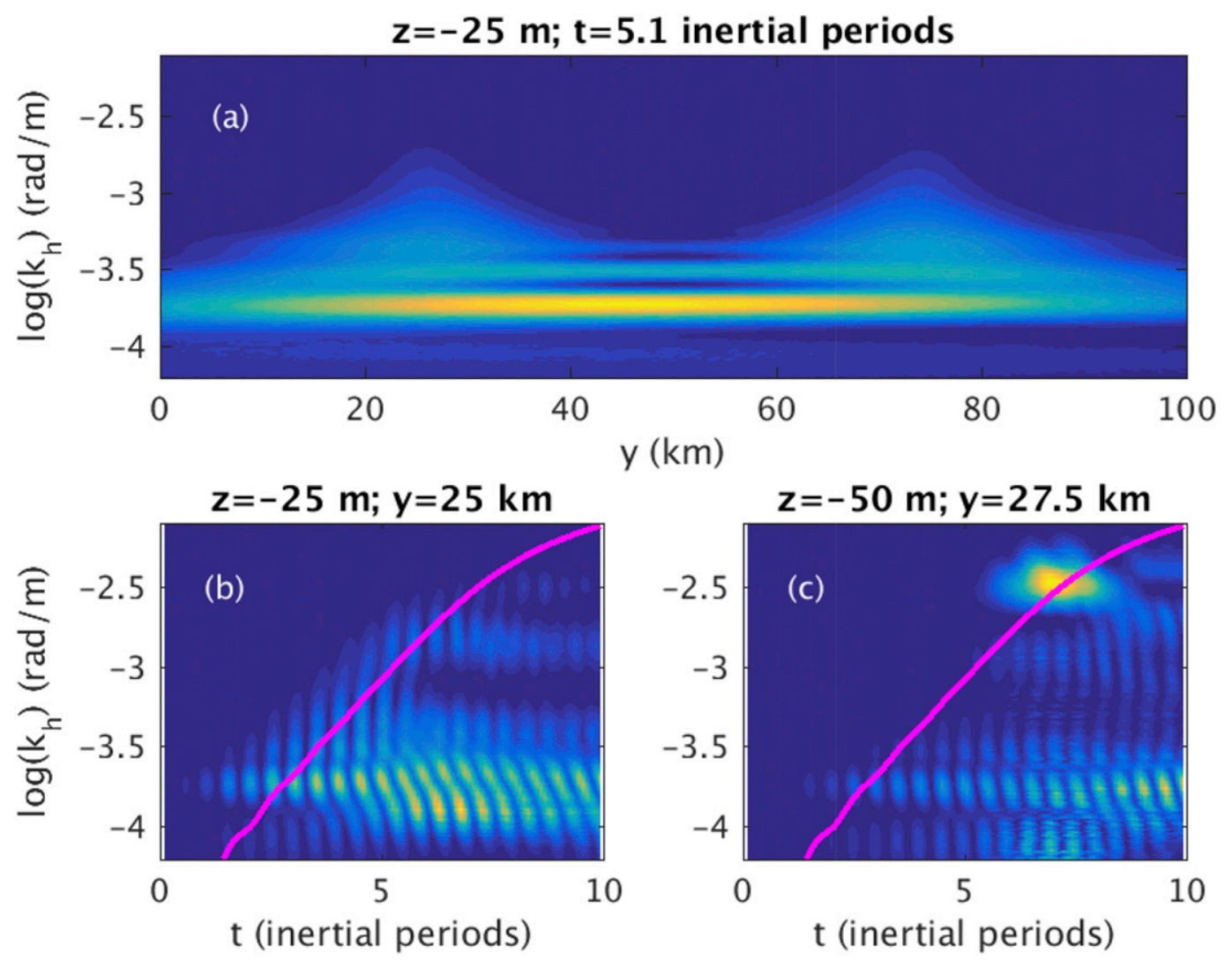

FIG. 5. Wavelet analysis to evaluate the evolution of the NIW's $y$ component of the wavevector in the proximity of the fronts for RUN1 and mode number $n=6$. (a) The square of the amplitude of the wavelet transform of the NIW's $v_{w}$ velocity (see Fig. 3) evaluated at $x=12.5 \mathrm{~km}, z=-25 \mathrm{~m}$, and $t=5.1$ inertial periods. (b) As in (a), but evaluated at $z=-25 \mathrm{~m}$ and $y=25 \mathrm{~km}$ (the center of the southern front) and plotted as a function of time. The magenta line is the theoretical prediction for the evolution the wavevector in (6) calculated at $z=-25 \mathrm{~m}$ and $y=25 \mathrm{~km}$. (c) As in (b), but evaluated at $z=-50 \mathrm{~m}$ and $y=25.5 \mathrm{~km}$ where a wave beam forms by $t \approx 7$ inertial periods (e.g., Fig. 3).

dominant horizontal wavenumber is smaller than those of the high modes. Presumably this is due to the stronger dependence of the group velocity in (2) on the vertical wavenumber relative to the horizontal wavenumber, which using (6) should scale as $c_{g, z} \propto n^{-1}$.

The formation of the wave beams in the simulations indicates that NIW energy is radiated into the stratified interior. The magnitude of this radiation can be quantified by calculating the vertical component of the energy flux areally integrated at a given level $z_{o}$ in the stratified interior:

$$
F_{e, z}=\left.\frac{1}{\mathscr{C}} \int_{\mathscr{b}} p_{w} w_{w}\right|_{z=z_{o}} d x d y
$$

The energy flux was evaluated at $z_{o}=-75 \mathrm{~m}$ over an area $A$ that covered the region between $0<y<100 \mathrm{~km}$ and $0<x<25 \mathrm{~km}$ (where the eddy driven strain field is frontogenetic) and its time evolution and dependence on mode number was assessed (Fig. 8). The behavior of $F_{e, z}$ for the simulation with and without fronts was contrasted by comparing the fluxes from RUN1 and RUN1-NF. For mode numbers greater than $n=1, F_{e, z}$ is stronger in the presence of fronts. The energy flux is downward and decreases with time, reaching a minimum value that depends on mode number. The magnitude of this minimum value plotted on a log scale versus mode number reveals that the energy flux follows different power laws in the presence and absence of fronts (Fig. 9). In the figure the energy flux has been normalized by a scaling for the energy flux associated with a NIW with mode number $n$ and a horizontal wavelength equal to the wavelength of the eddies $\lambda_{y}$, that is,

$$
F_{e, \text { scaling }}=\left(\frac{N_{\mathrm{ml}}^{2} H^{3} \rho_{o} u_{o}^{2}}{4 \pi f \lambda_{y}^{2}}\right) n^{-3},
$$

where the values of $u_{o}, \lambda_{y}, f$, and $N_{\mathrm{ml}}^{2}$ are listed in the appendix and Table 1 . With fronts, the normalized 

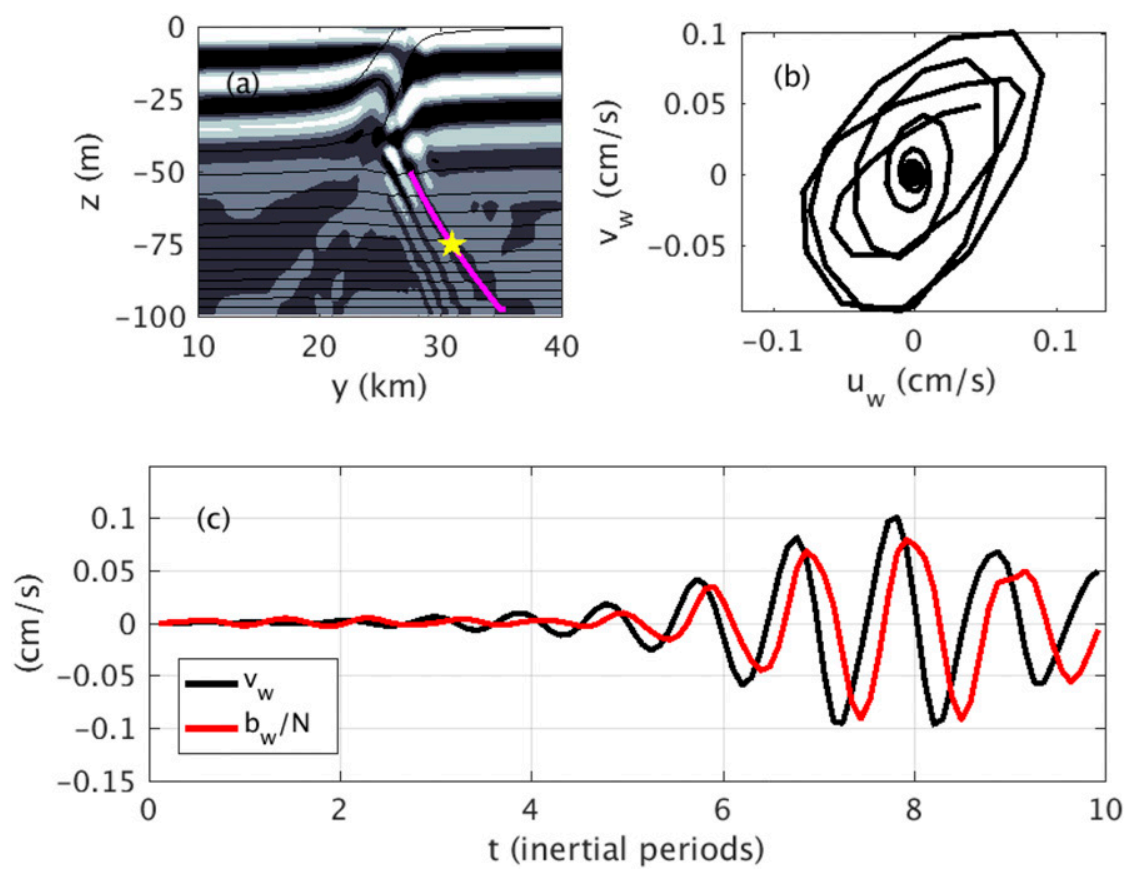

FIG. 6. (a) Same fields as in Fig. 3 at 7.1 inertial periods along with a ray path (magenta) for a wave with intrinsic frequency $\omega_{i}=1.7 f$. (b) Hodograph of the wave's velocity evaluated at the location indicated by the yellow star in (a). (c) Time series of the wave's meridional velocity $v_{w}$ (black) and buoyancy anomaly scaled by the inverse buoyancy frequency $b_{w} / N$ (red) at the same location as in (b).

energy flux follows a square power law, while without fronts it is nominally constant. This finding is consistent with the theoretical prediction for the vertical component of the group velocity discussed in section 2, verifying how Doppler shifting associated with frontal vertical circulations can greatly enhance the radiation of wave energy especially for high-mode NIWs.

The numerical experiments described thus far have explored the dependence of wave radiation on the vertical structure of the NIWs. In these simulations the nondimensional parameter $\delta=H_{w} / H_{b}=2 / n$ (using the vertical scale of the lateral buoyancy gradient $h=H / 2$, see the appendix, as a measure of $H_{b}$ ) varies between 2/7 and 2, suggesting that the Doppler shift is comparable in strength to refraction for the low modes but dominates for high-mode NIWs. Comparing the energy fluxes between the simulations run with and without fronts (e.g., Fig. 9) confirms this inference based on scaling arguments. Namely, for mode numbers greater than 3 , where $\delta<2 / 3, F_{e, z}$ is over an order of magnitude larger in the simulations with fronts.

\section{2) Dependence on Rossby number}

The other critical nondimensional parameter in the problem is the Rossby number $\varepsilon$, which is a measure of both the vertical vorticity and strain rate of the background eddy field. To study the dependence of wave radiation on the Rossby number, simulations with barotropic eddies of varying intensity were

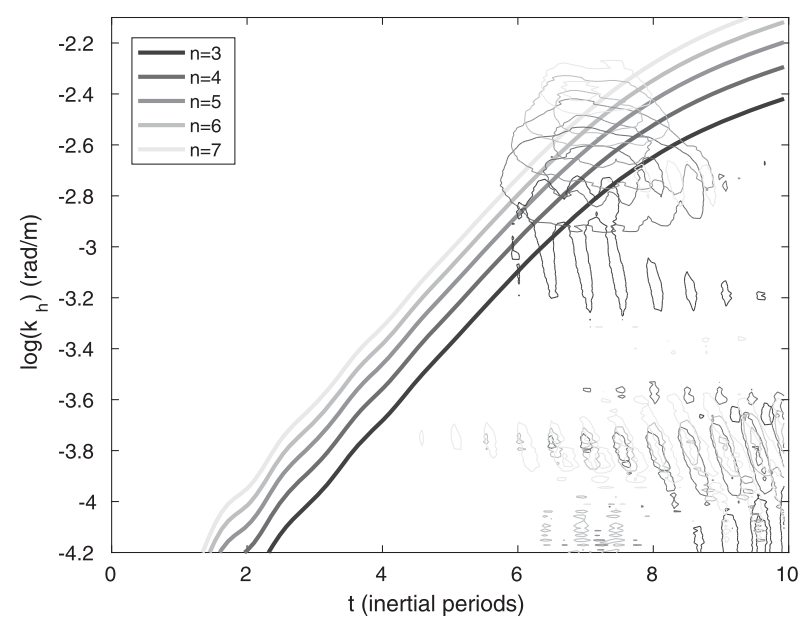

FIG. 7. As in Fig. 5c, but for NIWs initialized with different mode numbers, as indicated in the legend. The amplitude of the wavelet transform evaluated at $z=-50 \mathrm{~m}$ and $y=27.5 \mathrm{~km}$ (the location where a wave beam emanates from the southern front) is contoured and the thick lines are the theoretical prediction for the evolution the wavevector in (6). 


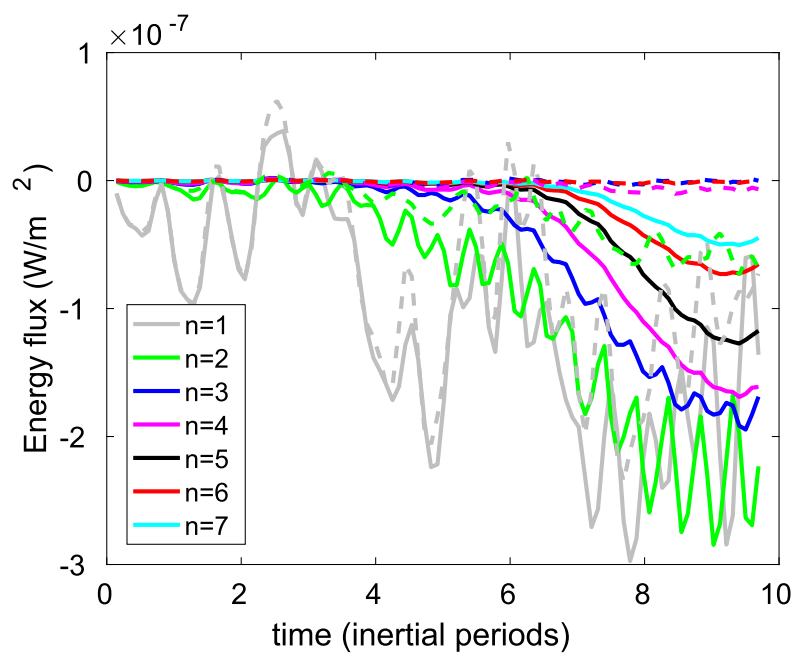

FIG. 8. Time series of the energy flux $F_{e, z}$ from simulations with fronts (i.e., RUN1, solid lines) and without fronts (i.e., RUN1-NF, dashed lines) for mode numbers $n$ between 1 and 7. Except for the lowest mode, $F_{e, z}$ grows more rapidly with fronts.

performed. The strength of the front and stratification were identical in all simulations and only the maximum strain rate $\alpha_{o}$, and hence Rossby number $\alpha_{o} / f$ of the eddies was varied. As before, two sets of simulations with and without fronts were run (see Table 1). Each simulation was initialized with an inertial oscillation with mode number $n=2$ and $\delta=1$. With $\delta=1$, the scalings (9) and (11) suggest that refraction should dominate over the vertical Doppler shift for lower Rossby numbers, while both processes should be equally important for higher Rossby numbers. However, time series of the energy flux reveal that by the end of the record, $F_{e, z}$ is similar in magnitude in the runs with and without fronts regardless of the value of the Rossby number (top panel of Fig. 10), implying that the waves in both types of simulations develop comparable lateral scales and vertical propagation speeds, more in line with the scaling (11). The temporal evolution of the energy flux is however quite different in the two sets of simulations, as the waves radiate energy more quickly when fronts are present. This is especially evident in the simulations with $\alpha_{o}=0.14 f$ (RUN3 and RUN3-NF), where $F_{e, z}$ reaches its minimum several inertial periods earlier in RUN3 versus RUN3-NF. The net effect in the upper part of the water column is a greater reduction in the wave's kinetic energy in simulations with fronts since the energy flux is active for a longer length of time. This is quantified by calculating the wave kinetic energy integrated over the upper $75 \mathrm{~m}$ and averaged areally over the same area $t 6$ used to calculate the energy flux in (13) (Fig. 10, bottom panel). The reduction in wave kinetic energy is larger for

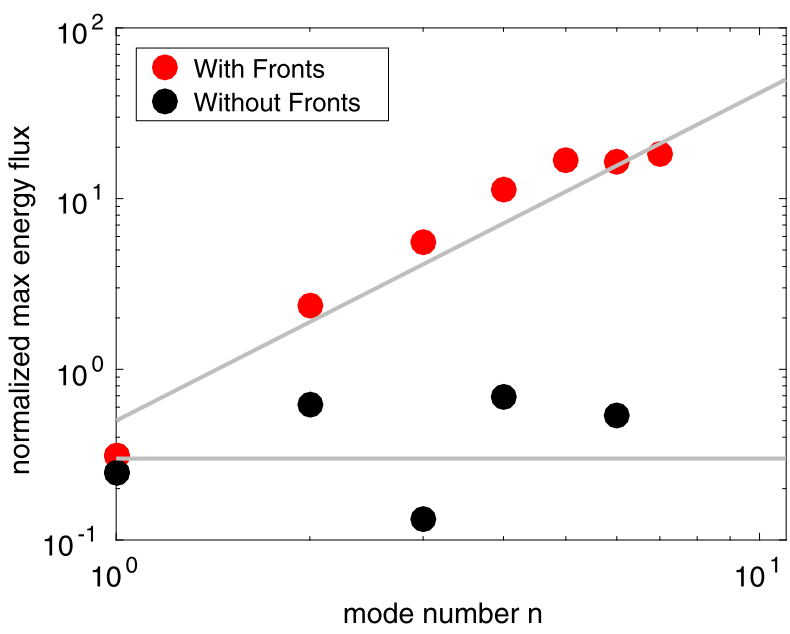

FIG. 9. Maximum with respect to time of the magnitude of the energy flux shown in Fig. 8 as a function of mode number for simulations with and without fronts. The energy flux has been normalized by a scaling for the energy flux in (14) associated with a NIW with the same mode number but with a lateral wavelength equal to the horizontal scale of the eddies. Lines with slopes of 2 and 0 are indicated and correspond to nonnormalized energy fluxes with an $\mathrm{m}^{-1}$ and $\mathrm{m}^{-3}$ dependence on the vertical wavenumber, respectively.

stronger strain rates and is enhanced in the presence of fronts. For example, for $\alpha_{o}=0.14 f$, by 7 inertial periods the change in wave $\mathrm{KE}$ is around $20 \%$ larger in RUN3 relative to RUN3-NF.
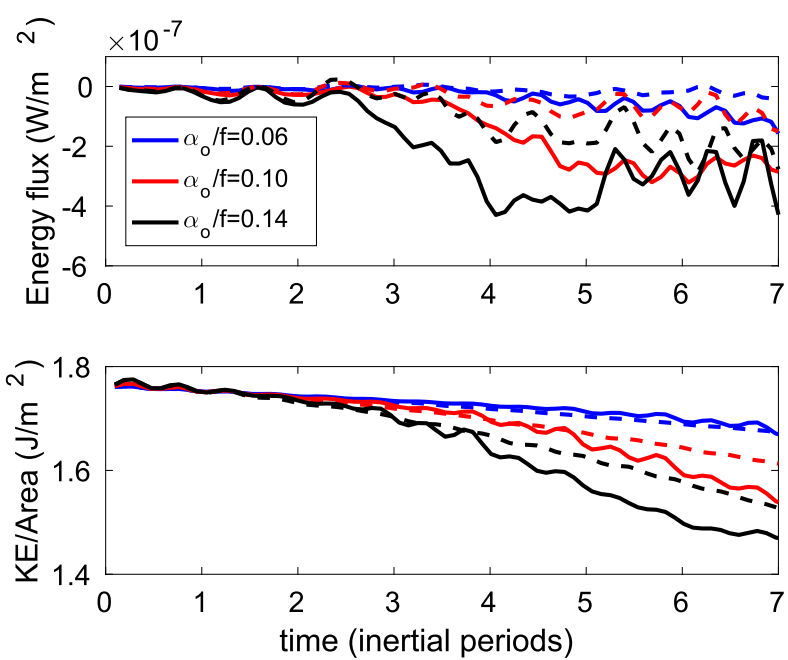

FIG. 10. (top) Time series of the energy flux $F_{e, z}$ evaluated at $z=-75 \mathrm{~m}$ from simulations with fronts (solid lines) and without fronts (dashed lines) for a NIW with mode number $n=2$ and for three values of the maximum strain rate $\alpha_{o}$. The initial value of the stratification and strength of the front (for simulations with fronts) is the same for all experiments. (bottom) Kinetic energy per unit area in the near-inertial wave field in the upper $75 \mathrm{~m}$ for the three sets of simulation with varying $\alpha_{o}$. 

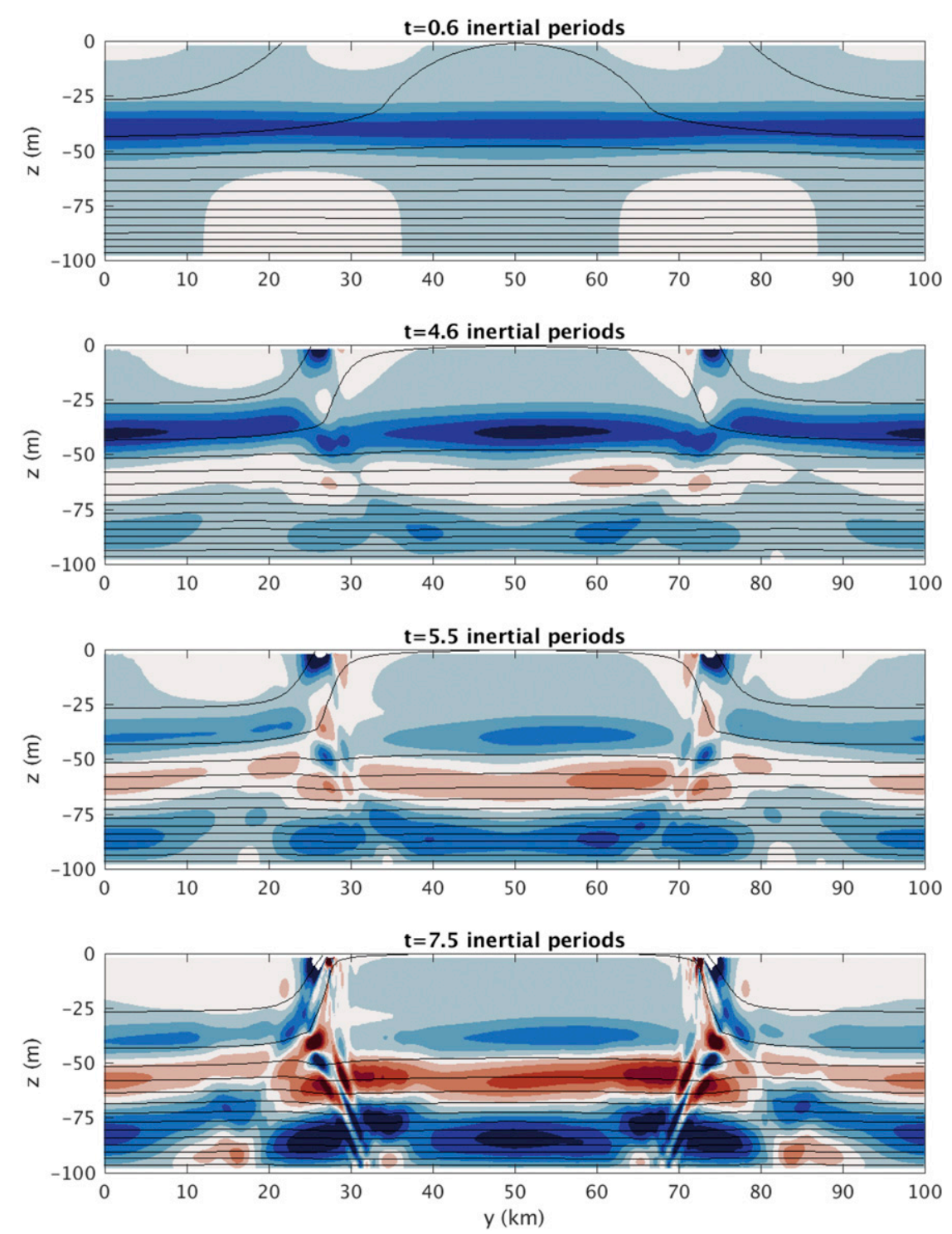

FIG. 11. Evolution of the shear in the wave field for the slab mixed layer run with fronts undergoing frontogenesis. The vertical shear of the $y$ component of the wave velocity is plotted in color, and isopycnals are contoured at $x=12.5 \mathrm{~km}$. The maximum wave shear is $3 \times 10^{-4} \mathrm{~s}^{-1}$.

\section{c. Simulations with slab-layer wave forms}

The idealized wave forms used in the numerical experiments described above yield results that can be more easily compared to the theoretical prediction for the horizontal wavenumber (6) since they are characterized by a single, dominant vertical wavenumber, see, (A5). To explore the effects of the Doppler shift in a more realistic wave field, a wave form emulating a slab-layer inertial oscillation with velocity $v_{w}=0$ and

$$
u_{w}=\frac{u_{o}}{2}\left[1+\tanh \left(\frac{z+h_{v}}{L_{z}}\right)\right]
$$

was used as an initial condition (where $h_{v}=40 \mathrm{~m}$, $L_{z}=10 \mathrm{~m}$, and $u_{o}=0.01 \mathrm{~m} \mathrm{~s}^{-1}$ ). As before, simulations with and without fronts were contrasted to highlight differences in the wave dynamics caused by the Doppler shift.

Similar to the idealized wave forms, waves with a slab-layer initial condition develop finer lateral scales when fronts are present (cf. Figs. 11 and 12 ). On larger scales, however, the waves in the simulations with and without fronts share common features. These features are associated with low-mode NIWs that carry most of the energy in a slab-layer-like initial condition (e.g., Gill 1984). Low-mode NIWs are less affected by the 

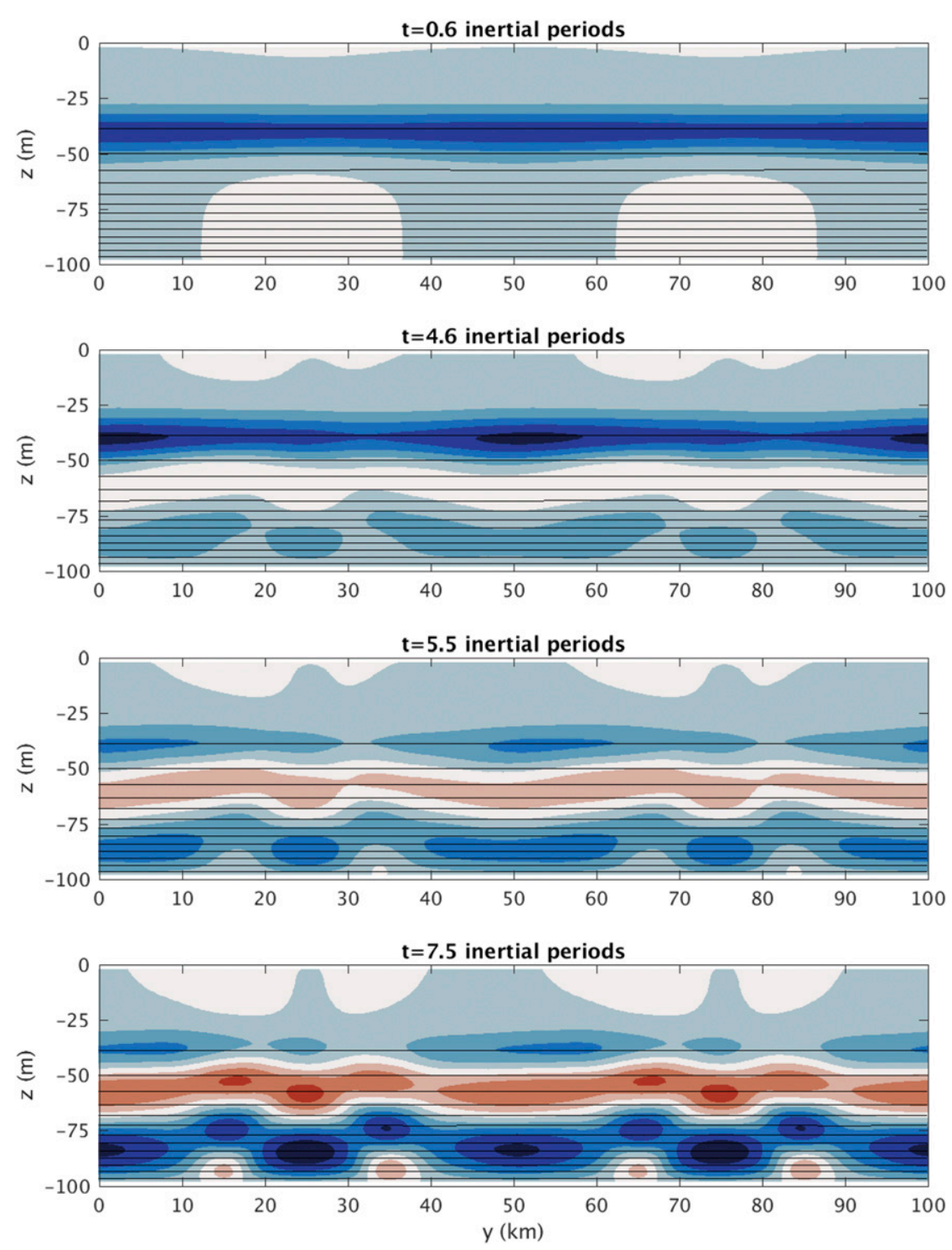

FIG. 12. As in Fig. 11 for a simulation with the same eddy field, but without the fronts.

Doppler shift (e.g., Fig. 9), explaining why their evolution is nearly identical in the two types of simulations. The high-mode NIWs are however affected by the frontal vertical circulation and tend to radiate energy away from the fronts in beams that form on both sides of the fronts. Ray tracing was used to understand how this pattern of wave propagation is established. A ray with intrinsic frequency $\omega_{i}=1.8 f$ emanating from a single location appears to explain the two beams (Fig. 13). The location is on the dense side of the front at a depth of $40 \mathrm{~m}$. This is where the shear in the initial condition in (15) is largest and frontal downwelling extends to greater depths than upwelling (e.g., Fig. 2, bottom panel), thus maximizing vertical advection and the Doppler shift. It appears that energy radiates from this location in both directions along the ray path. In particular, an upward propagating wave transits the mixed layer and reflects off the surface, returning back into the stratified interior and toward the light side of the front following the ray trajectory and beam. Time series of the wave velocity and buoyancy anomalies within the beam (not shown) reveal that the Eulerian frequency of the waves is close to inertial while the ratio of wave potential to kinetic energy is consistent with the superinertial intrinsic frequency $\omega_{i}=1.8 f$ inferred from ray tracing. A Doppler shift associated with the barotropic velocity of the eddy field can account for this difference between Eulerian and intrinsic frequencies, as was found in the simulations with the idealized wave forms (see section 3b). 


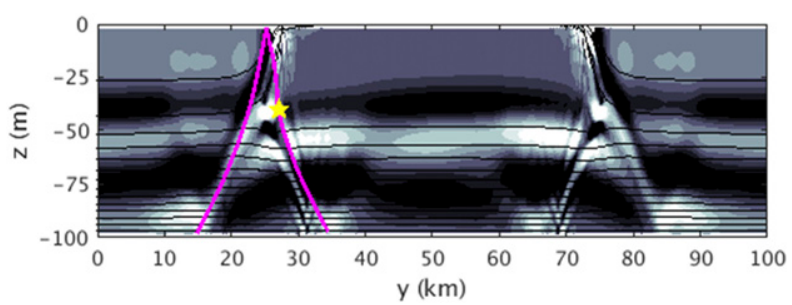

FIG. 13. As in Fig. 11, but evaluated at 8.6 inertial periods and with rays (magenta lines) corresponding to the paths traveled by wavepackets with intrinsic frequency $1.8 f$ fired both upward and downward at the location indicated by the yellow star.

Initializing the simulations with a slab-layer wave form is a simple way to represent wind-forced inertial motions without the forcing. The findings from these simulations indicate that such waves experience a more complex interaction with vertical circulations than the idealized wave forms and highlight the importance of the vertical collocation of the inertial shear and upwelling/downwelling. This suggests that NIWs that are actively being forced by winds will experience similar complex behavior at fronts, with potential implications for both the generation and propagation of the waves. Studying the effects of vertical Doppler shifting on forced NIWs is thus motivated and should be the focus of future work.

\section{Conclusions}

The dynamics of high-mode NIWs is not well understood. The small vertical scales of the high modes suggest that they should be susceptible to Doppler shifting by vertical motions. While Doppler shifting by vertical velocities associated with the internal wave continuum has been considered and is thought to smear NIW shear signals across a wide range of frequencies (e.g., Kunze et al. 1990; Sherman and Pinkel 1991; Pinkel 2014), Doppler shifting by frontal vertical circulations has not been explored. Here it is shown that the process can lead to Doppler shifts that are a significant fraction of the inertial frequency. They also introduce lateral phase differences in the wave field and hence enhance vertical radiation. If the frontal vertical motions are driven by frontogenetic strain, the development of lateral phase differences is an exponentially fast process and leads to the rapid radiation of NIW beams away from the front and down into the stratified interior. The mechanism results in a reduction of NIW kinetic energy in the upper ocean that increases with the rate of strain. In addition, it can enhance the magnitude of NIW shear (cf. Figs. 11 and 12) and thus could facilitate the dissipation of NIW energy through the generation of turbulence.
Doppler shifting by frontal vertical motions is a mechanism for sharpening the lateral scales of NIWs which is distinct from the traditional explanation involving refraction associated with gradients in vertical and planetary vorticity (D'Asaro 1989; Young and Ben-Jelloul 1997; van Meurs 1998). In particular, the development of lateral phase differences by vertical Doppler shifting depends on the vertical wavenumber $m$ of the NIWs, unlike refraction. As a result, the downward flux of NIW energy out of the upper ocean scales as $m^{-1}$ with Doppler shifting, versus $\mathrm{m}^{-3}$ with refraction (e.g., Fig. 9). Therefore high-mode NIWs are much more effective at radiating energy in an eddy field when frontal vertical circulations are present. In this context, high-mode specifically refers to waves with small vertical scales relative to the depth of the frontal vertical circulation.

The beams of NIWs that emanate from fronts through vertical Doppler shifting have characteristics of superinertial waves yet Eulerian frequencies very near $f$. In particular, the waves' current ellipses are far from circular and their potential energy (PE) and kinetic energy (KE) are comparable in magnitude, implying that their intrinsic frequency is superinertial (e.g., Fig. 6). This apparent discrepancy is explained by a Doppler shift, which in this case is associated with the horizontal rather than vertical component of the background flow. The short horizontal wavelengths in the beam combined with the confluent flow of the barotropic eddies yield a Doppler shift that keeps the Eulerian frequency near its initial value $f$ while allowing the intrinsic frequency to be superinertial. Conservation of Eulerian frequency might explain this phenomenon. According to ray theory, if the medium in which a wave propagates does not change in time, the Eulerian frequency is conserved along a ray (Gill 1982). Beneath the fronts, the stratification, background currents, and hence wave medium experience minimal changes in time. Therefore inertial motions should conserve their Eulerian frequency as they are converted to downward-propagating internal waves by vertical Doppler shifting. Similar internal waves with near-inertial Eulerian frequencies and elevated PE to KE ratios have been observed in the ocean but their generation has been a mystery (e.g., Sherman and Pinkel 1991; Pinkel 2014). The interaction of inertial motions and fronts undergoing frontogenesis, as described here, is one mechanism that could explain their formation.

Shear associated with high-mode NIWs is thought to play an important role in driving mixing and deepening of the mixed layer, with implications for climate 
(Jochum et al. 2013). Therefore parameterizing the energetics of these waves in coarse-resolution models, in particular their vertical radiation out of the mixed layer is well motivated. The theory and numerical simulations described in this article suggest that the physics of vertical Doppler shifting should be taken into account when parameterizing the damping of highmode NIWs in the upper ocean since the process can greatly enhance their energy fluxes. Developing such a parameterization presents challenges given the submesoscale nature of the dynamics involved. However, based on the numerical experiments describe here, it is clear that there are a few key factors that should be taken into consideration, namely the distribution of the vertical scales of the waves and the fronts, and the strength of the mesoscale strain and lateral density gradients. Having said this, there are certain characteristics of the flow used in the experiments that are quite idealized, and more work should be done in studying vertical Doppler shifting in a more realistic setting before formulating and implementing a parameterization of its effects. In particular, the confluence associated with the eddies that drives the frontal vertical circulations in the simulations does not vary in time and thus results in a persistent and strengthening vertical Doppler shift. If the eddies and fronts were more transient, the interaction of the NIWs with the frontal vertical circulation may not be as effective. Investigating vertical Doppler shifting in simulations with an evolving, turbulent, baroclinic eddy field and with inertial motions forced by winds (rather than imposed as an initial condition) are especially needed, and will be the subject of future research.

Acknowledgments. This research was supported by the Office of Naval Research Award N00014-18-1-2798.

\section{APPENDIX}

\section{Initial Conditions for Numerical Simulations}

The numerical experiments are configured with surface-intensified fronts and a pycnocline. They are initialized with a lateral buoyancy gradient that alternates sign sinusoidally in the $y$ direction and that decays with depth

$$
-\frac{\partial b_{i}}{\partial y}=\frac{S_{o}^{2}}{2}\left[1+\tanh \left(\frac{z+h}{\lambda}\right)\right]^{2} \sin \left(\frac{2 \pi y}{L_{y}}\right),
$$

and a stratification that increases linearly with depth below a certain level $z=-h_{o}$ :

$$
\frac{\partial b_{i}}{\partial z}=\left\{\begin{array}{cc}
N_{\mathrm{ml}}^{2}, & z>-h_{o}, \\
N_{\mathrm{ml}}^{2}-\frac{2 N_{\mathrm{ml}}^{2}}{\delta_{s}}\left(z+h_{o}\right), & z \leq-h_{o},
\end{array}\right.
$$

where $L_{y}$ is the width of the domain in the $y$ direction and $S_{o}^{2}, N_{\mathrm{ml}}^{2}, \lambda, h_{o}$, and $\delta_{s}$ are constants. The front in (A1) is accompanied by a geostrophic flow $u_{g, i}$ that satisfies the thermal wind balance and that has no barotropic flow, that is, $\int_{-H}^{0} u_{g, i} d z=0$, where $H$ is the depth of the numerical domain. Strain is provided by an array of geostrophic, barotropic eddies with velocity

$$
\begin{aligned}
& v_{e}=\frac{\alpha_{o} \lambda_{y}}{2 \pi} \sin \left(\frac{2 \pi x}{\lambda_{x}}\right) \sin \left(\frac{2 \pi y}{\lambda_{y}}\right), \\
& u_{e}=\frac{\alpha_{o} \lambda_{x}}{2 \pi} \cos \left(\frac{2 \pi x}{\lambda_{x}}\right) \cos \left(\frac{2 \pi y}{\lambda_{y}}\right),
\end{aligned}
$$

where $\alpha_{o}$ is the maximum strain rate, and $\lambda_{x}$ and $\lambda_{y}$ are the wavelengths of the eddies in the $x$ and $y$ direction, respectively, which are both set to half the domain width in the $y$ direction, that is, $\lambda_{x}=\lambda_{y}=L_{y} / 2$. Several of the constants in (A1) and (A2) are fixed for all of the numerical experiments, namely $\lambda=25 \mathrm{~m}$, $h=50 \mathrm{~m}, h_{o}=30 \mathrm{~m}$, and $\delta_{s}=10 \mathrm{~m}$. The parameters that vary are listed in Table 1.

A NIW is added to the background flow field as an initial condition. It has a wave form with the following structure

$$
\left(\begin{array}{c}
u_{w} \\
v_{w}
\end{array}\right)=\frac{u_{o}}{2}\left(\begin{array}{c}
\cos m z \\
\sin m z
\end{array}\right)\left[1+\tanh \left(\frac{z+h_{v}}{L_{z}}\right)\right],
$$

where $\left(u_{w}, v_{w}\right)$ is the wave velocity at $t=0$ with speed $u_{o}$, which is a plane wave with wavenumber $m$ modulated in the vertical by the function in the square brackets that confines the NIW to a layer near the surface of depth $h_{v}$ and decays in the vertical over a length scale $L_{z}$. In the experiments $m$ is varied, but the parameters of the envelope are fixed, that is, $h_{v}=40 \mathrm{~m}$ and $L_{z}=10 \mathrm{~m}$, as well as the speed, $u_{o}=0.01 \mathrm{~m} \mathrm{~s}^{-1}$.

\section{REFERENCES}

Alford, M. H., J. A. MacKinnon, H. L. Simmons, and J. D. Nash, 2016: Near-inertial internal gravity waves in the ocean. Annu. Rev. Mar. Sci., 8, 95-123, https://doi.org/10.1146/ annurev-marine-010814-015746.

D'Asaro, E. A., 1989: The decay of wind-forced mixed layer inertial oscillations due to the $\beta$ effect. J. Geophys. Res., 94, 2045-2056, https://doi.org/10.1029/JC094iC02p02045.

_ 1995: Upper-ocean inertial currents forced by a strong storm. Part III: Interaction of inertial currents and mesoscale eddies. 
J. Phys. Oceanogr., 25, 2953-2958, https://doi.org/10.1175/ 1520-0485(1995)025<2953:UOICFB >2.0.CO;2.

Gill, A. E., 1982: Atmosphere-Ocean Dynamics. International Geophysics Series, Vol. 30, Academic Press, 662 pp.

_ 1984: On the behavior of internal waves in the wakes of storms. J. Phys. Oceanogr., 14, 1129-1151, https://doi.org/ 10.1175/1520-0485(1984)014<1129:OTBOIW>2.0.CO;2.

Hoskins, B. J., I. Draghici, and H. C. Davies, 1978: A new look at the $\omega$-equation. Quart. J. Roy. Meteor. Soc., 104, 31-38, https:// doi.org/10.1002/qj.49710443903.

Jochum, M., B. Briegleb, G. Danabasoglu, W. Large, N. Norton, S. Jayne, M. Alford, and F. Bryan, 2013: The impact of oceanic near-inertial waves on climate. J. Climate, 26, 2833-2844, https://doi.org/10.1175/JCLI-D-12-00181.1.

Kunze, E., M. G. Briscoe, and A. J. Williams, 1990: Interpreting shear and strain finestructure from a neutrally buoyant float. J. Geophys. Res., 95, 18111-18125, https://doi.org/10.1029/ JC095iC10p18111.

Pedlosky, J., 1987: Ageostrophic motion. Geophysical Fluid Dynamics, Springer, 624-688.

Pinkel, R., 2014: Vortical and internal wave shear and strain. J. Phys. Oceanogr., 44, 2070-2092, https://doi.org/10.1175/ JPO-D-13-090.1.

Pollard, R. T., and L. A. Regier, 1992: Vorticity and vertical circulation at an ocean front. J. Phys. Oceanogr., 22, 609-625, https://doi.org/10.1175/1520-0485(1992)022<0609:VAVCAA $>$ 2.0.CO;2.

Rocha, C. B., G. L. Wagner, and W. R. Young, 2018: Stimulated generation: Extraction of energy from balanced flow by near-inertial waves. J. Fluid Mech., 847, 417-451, https:// doi.org/10.1017/jfm.2018.308.

Rudnick, D. L., 1996: Intensive surveys of the Azores Front. 2. Inferring the geostrophic and vertical velocity fields. J. Geophys. Res., 101, 16-291-16303, https://doi.org/10.1029/96JC01144.

Shchepetkin, A. F., and J. C. McWilliams, 2005: The Regional Ocean Modeling System (ROMS): A split-explicit, freesurface, topography-following coordinate oceanic model. Ocean Modell., 9, 347-404, https://doi.org/10.1016/ j.ocemod.2004.08.002.

Sherman, J. T., and R. Pinkel, 1991: Estimates of the vertical wavenumber-frequency spectra of vertical shear and strain. J. Phys. Oceanogr., 21, 292-303, https://doi.org/10.1175/15200485(1991)021<0292:EOTVWS > 2.0.CO;2.

Thomas, L. N., A. Tandon, and A. Mahadevan, 2008: Submesoscale processes and dynamics. Ocean Modeling in an Eddying Regime, Geophys. Monogr., Vol. 177, Amer. Geophys. Union, 17-38.

— C. M. Lee, and Y. Yoshikawa, 2010: The subpolar front of the Japan/East Sea II: Inverse method for determining the frontal vertical circulation. J. Phys. Oceanogr., 40, 3-25, https:// doi.org/10.1175/2009JPO4018.1.

van Meurs, P., 1998: Interactions between near-inertial mixed layer currents and the mesoscale: The importance of spatial variabilities in the vorticity field. J. Phys. Oceanogr., 28, 1363-1388, https:// doi.org/10.1175/1520-0485(1998)028<1363:IBNIML > 2.0.CO;2.

Young, W. R., and M. Ben-Jelloul, 1997: Propagation of nearinertial oscillations through a geostrophic flow. J. Mar. Res., 55, 735-766, https://doi.org/10.1357/0022240973224283. 